\title{
26
}

\section{DESTINO CANADÁ: VISIÓN GENERAL Y PERSPECTIVAS DEL PAÍS}

Canadá es un país de gran extensión, muy rico en recursos naturales (madera, petróleo, gas y minerales), con un sector agrícola potente centrado en el cultivo de cosechas y con un desarrollo industrial elevado, destacando los sectores de automoción, aeronáutica, alimentación o telecomunicaciones.

Dada su orientación al exterior, su política comercial ha venido marcada por la búsqueda de reglas claras y estables tanto a nivel multilateral como bilateral, lo que se ha traducido en la firma de numerosos acuerdos comerciales, entre ellos con la Unión Europea. Estos últimos no han logrado reducir la significativa dependencia que mantiene con Estados Unidos.

A pesar de haberse visto fuertemente afectado por la crisis derivada de la COVID-19, las perspectivas económicas de Canadá son favorables, sobre todo, en la medida en que haga frente a sus principales desafíos: el avance hacia un mercado interior único, el impulso de la competencia, la transición energética y la mejora de sus infraestructuras.

Palabras clave: Canadá, sectores económicos, crecimiento económico, política comercial.

Clasificación JEL: E66, F13, O51.

\section{Introducción}

Canadá y España comparten valores fundamentales como la construcción de sociedades inclusivas, la economía de mercado, la defensa de una economía abierta o la lucha contra el cambio climático. Sin embargo, y aunque la relación comercial entre Canadá y España se remonta al siglo xvi, con la presencia de balleneros vascos en Labrador y Terranova, se trata de un país, en general, poco conocido por las empresas españolas.

\footnotetext{
* Consejera Económica y Comercial Jefe de la Oficina Económica y Comercial en Ottawa.

Versión de agosto de 2021.

DOI: https://doi.org/10.32796/bice.2021.3140.7282
}

Efectivamente, no es de los primeros destinos incluidos por parte de una empresa española en sus decisiones preliminares de internacionalización debido al uso de un idioma distinto y, sobre todo, a que otros países en la región presentan claras ventajas en términos de un mayor mercado y mayor presencia de empresas españolas. No obstante, se trata de un país de gran estabilidad política, con alto nivel de renta y con instituciones sólidas que garantizan la transparencia y el cumplimiento de unas reglas de juego estables, que ofrece importantes oportunidades a las empresas españolas.

Con vistas a ofrecer una primera aproximación a las empresas poco familiarizadas con $\triangleright$ 
el país, el presente artículo recoge una visión general de la economía de Canadá, de su política comercial, así como de las perspectivas futuras, incluyendo los principales retos que tiene por delante.

\section{Visión general de los principales sectores productivos de Canadá}

Si bien la estructura económica de Canadá es similar a la de otros países con alto nivel de renta - con un sector servicios que supera el $70 \%$ del PIB, un sector industrial en el entorno del $26-27 \%$ y un sector agropecuario, incluyendo silvicultura y pesca, que supone el 2,2$2,4 \%$ - cuando se analizan los componentes de las exportaciones se observa la gran riqueza del país en recursos naturales, en particular en madera, petróleo, gas y minerales.

Así, el sector forestal se ha caracterizado por un desarrollo sostenido, siendo sus principales productos el papel prensa y la madera blanda, y sus exportaciones en 2020 tuvieron un peso por encima del $8 \%$ del total de exportaciones. Canadá posee el $10 \%$ de la superficie de bosques del mundo y es el segundo productor de madera blanda.

Aunque importa un volumen considerable de alimentos elaborados, Canadá es uno de los principales exportadores de productos agropecuarios y pesqueros del mundo, que suponen el $8,41 \%^{1}$ del total de sus exportaciones. Destacan el cultivo de cosechas (trigo, colza, cebada y otros granos) muy orientado a la exportación; los mariscos y productos derivados, y la ganadería de vacuno y de porcino.

Este dato incluye también los productos alimenticios intermedios. https://www150.statcan.gc.ca/t1/tbl1/en/tv.action?pid=1210013001
Finalmente, el sector extractivo ha jugado y sigue jugando un papel determinante en el desarrollo del país. Por un lado, Canadá es uno de los principales países mineros y explota la extracción de más de sesenta minerales y metales. Estos últimos representan el $50 \%$ del total de la producción. Canadá es el primer productor de potasa en el mundo, el segundo de uranio y niobio, y se sitúa entre los cinco primeros países en la producción de otros catorce minerales y metales, tales como níquel, cobalto, diamantes, gemas, oro, grafito, indio, cadmio, platino o titanio. Con una contribución al PIB del $5 \%$ en 2019, el sector minero emplea a más de 700.000 trabajadores directos e indirectos y concentra el $19 \%$ de las exportaciones canadienses (15,6\% en 2020).

La importancia del sector minero canadiense tiene una vertiente internacional muy marcada. La Bolsa de Valores de Toronto -en la que cotizan el $48 \%$ de las empresas mineras y de exploración-es la principal a nivel mundial en este sector. En 2019 canalizó la mitad del capital obtenido en el mundo para proyectos mineros. Además, del total de activos de explotación y exploración que las empresas canadienses poseen (unos 180.000 millones de euros), unos dos tercios se encuentran situados en el exterior, en particular en América Latina y Caribe, que acoge el $46,5 \%$ de los activos mineros canadienses en el extranjero.

A futuro, las prioridades de las autoridades canadienses se centran en mejorar la resiliencia de las cadenas de valor en cuanto a minerales críticos - los necesarios para las tecnologías limpias, incluidas las baterías- alcanzando acuerdos con socios que garanticen la seguridad del abastecimiento. En este sentido, Canadá tiene ya firmado un plan de acción con Estados Unidos y tiene abierto un diálogo con la Unión Europea y con Japón. 
Por otro lado, Canadá es el cuarto productor mundial de petróleo y gas y acumula importantes reservas. En los últimos treinta años, Canadá ha pasado de producir 1,7 millones de barriles de petróleo bruto al día (Mbd) a producir 4,7 Mbd en 2019. Hasta principios de los años 2000 , el crecimiento se concentró en el petróleo clásico (pesado y ligero) y condensados, pasando el protagonismo, a partir de 2009, a los yacimientos de las arenas bituminosas de Alberta.

Los precios elevados del petróleo a finales de los años 2000 y hasta 2015 favorecieron la intensificación de la producción y un gran crecimiento de las exportaciones de petróleo bruto canadiense, que se multiplicaron por 15 entre 1990 y 2019, suponiendo en esta última fecha el $15,27 \%$ de las exportaciones totales. A pesar de disponer de las terceras mayores reservas a nivel mundial, el sector petrolífero canadiense se ve muy condicionado por las dificultades de construir nuevos oleoductos —que han alcanzado el máximo de capacidad-dirigidos hacia EE. UU., mercado que absorbió el $97 \%$ del total de las exportaciones de crudo en 2019.

Pasando a considerar el sector secundario, hay que empezar mencionando un cierto declive del sector manufacturero desde principios del siglo XXI, cuando aportaba casi un $16 \%$ del PIB. El sector acusó una grave crisis en 2008-2009, de la que ha ido recuperándose, aunque sin alcanzar los niveles de finales del siglo $x x$, pues aporta en el entorno del $10-11 \%$ al PIB. Distinta evolución ha conocido el empleo manufacturero, con una pérdida continua del orden de 400.000 empleos desde el año 2000. No obstante, esta tendencia se ha revertido en los últimos años coincidiendo con un importante crecimiento de la industria de la alimentación - sobre todo cárnica- muy intensiva en trabajo.
Aparte de la industria de la alimentación, destaca sin duda el sector de la automoción, localizado en la zona de los Grandes Lagos y plenamente integrado con el estadounidense. Canadá se encuentra entre los diez principales productores de vehículos ligeros del mundo y alberga diversas plantas de cinco fabricantes estadounidenses y japoneses: Fiat-Chrysler, Ford, General Motors, Honda y Toyota. Adicionalmente, refuerzan el sector más de setecientas empresas de autopartes.

La producción canadiense de vehículos alcanzó un pico de 3 millones de unidades en 1999. A pesar de cierta recuperación a principios de la década de 2010, con producciones anuales de 2,4 millones, el declive ha sido continuado -coincidiendo con la desaceleración económica de EE. UU. y una pérdida de competitividad del sector $-y$, actualmente, la fabricación anual de vehículos se sitúa entre 1,7 y 1,9 millones de unidades.

Se trata de un sector muy orientado a la exportación, con el $85 \%$ de los vehículos ensamblados en Canadá exportados a Estados Unidos. Tradicionalmente, el comercio bilateral ha sido superavitario para Canadá en vehículos y deficitario en autopartes, superando el saldo de los vehículos al de autopartes y arrojando una balanza comercial positiva para Canadá. La caída en la producción de unidades en Canadá, con la consiguiente menor exportación, así como el incremento de las importaciones provenientes de EE. UU., México y la Unión Europea, han ido arrastrando el saldo hacia un déficit que se prevé continúe a futuro.

En contraposición al declive mencionado anteriormente, los diversos anuncios realizados desde finales de 2020 por parte de Ford, Fiat-Chrysler, General Motors y Toyota de ensamblar vehículos híbridos o completamente eléctricos en sus plantas de Canadá, así $\square$ 
como la apuesta por el vehículo eléctrico de los grandes Tier- 1 canadienses como Magna o Dana, arrojan optimismo de cara al futuro del sector. Estos planes, apoyados por fondos federales y provinciales, se verán también impulsados por el lado de la demanda gracias a los generosos incentivos que las principales provincias ofrecen a los compradores de vehículos eléctricos.

Otros sectores manufactureros también relevantes en Canadá son las industrias de alta tecnología, en particular las telecomunicaciones, la industria aeronáutica y aeroespacial, la industria de la informática y los equipos médicos.

Por último, el sector servicios aporta el $71 \%$ del PIB y emplea al $79 \%$ de los trabajadores -incluyendo las Administraciones públicas-, siendo los mayores empleadores los sectores del retail y de servicios comerciales. Aunque los servicios inmobiliarios representan el subsector más importante, destacan por su dinamismo reciente: los servicios profesionales, científicos y técnicos; los servicios educativos; las industrias culturales y de la información; y los servicios de salud y el turismo, si bien este último se ha visto muy negativamente impactado por la COVID-19.

Canadá tiene uno de los sectores de servicios financieros más sólidos del mundo, que ocupa el noveno lugar entre 137 economías en cuanto al desarrollo del mercado financiero, según el Informe sobre la Competitividad Mundial 2019 del Foro Económico Mundial. Con una aportación al PIB del 7,6\% en 2020 y más de 900.000 empleos, es uno de los sectores que más contribuyen a la economía. Se trata de un sector relativamente concentrado, en el que, a finales de 2020, los cinco grandes bancos nacionales gestionaban 7,3 billones de dólares canadienses, es decir, el $87 \%$ de los activos bancarios totales, y en el que más del
$90 \%$ de los activos de compañías de seguros de vida y salud pertenecen a las cinco mayores empresas nacionales.

\section{Política comercial de Canadá}

\subsection{Canadá, defensor del multilateralismo}

No es de extrañar que Canadá, con un potente sector industrial, importantes recursos naturales y un mercado doméstico reducido (38 millones de habitantes), tenga una economía orientada al exterior. A modo ilustrativo, la población canadiense representa el $0,5 \%$ de la población mundial y sus exportaciones alcanzan casi el $2,5 \%$ de las exportaciones mundiales de mercancías. En 2019, los flujos comerciales de mercancías alcanzaron un máximo histórico superior a los 800.000 millones de euros.

El acceso a los mercados extranjeros es, por tanto, fundamental para Canadá, lo que explica su activo papel en la promoción del multilateralismo y del buen funcionamiento de la Organización Mundial del Comercio, así como la prioridad que las autoridades canadienses han otorgado a dotar a las relaciones comerciales con Estados Unidos, su principal socio comercial, de unas reglas estables que promuevan los flujos de comercio e inversión. Así, en 1985, ambos países iniciaron las negociaciones de un acuerdo de libre comercio Canadá-EE. UU. que entró en vigor el 1 de enero de 1989 y que estuvo vigente hasta el 1 de enero de 1994, fecha de la entrada en vigor del Tratado de Libre Comercio de América del Norte (NAFTA, por sus siglas en inglés), que incluye también a México.

En este contexto, la integración de la economía canadiense en el tejido económico de $\triangleright$ 
Estados Unidos se intensificó enormemente, llegando a suponer las exportaciones canadienses a su vecino del sur el $87 \%$ del total de sus exportaciones en 2002. Si bien esta dependencia ha ido reduciéndose, la entrada en vigor, en julio 2020, del Acuerdo entre Canadá, EE. UU. y México (CUSMA, por sus siglas en inglés), con nuevas normas de origen que refuerzan el contenido de valor regional para los fabricantes de vehículos, tejidos, químicos, hierro y acero, permite anticipar que la relación comercial y de inversión entre los tres países firmantes continuará estrechándose en el futuro.

Cabe señalar asimismo que, si bien la firma del CUSMA fue decisiva para dejar atrás unos años de incertidumbre y conflictividad en la relación comercial entre EE. UU. y Canadá, su entrada en vigor no ha supuesto la desaparición de determinados focos de preocupación para Canadá, entre los que cabe mencionar la apertura de un proceso de consultas por parte de EE. UU. en relación con la gestión de cuotas que hace Canadá de los productos lácteos; la imposición de aranceles por parte de EE. UU. a la madera blanda y a los equipos de energía solar canadienses; o el programa «Buy America» del presidente Biden, que puede dificultar seriamente la participación de las empresas canadienses en el plan de renovación de infraestructuras de Estados Unidos.

\subsection{A la búsqueda de diversificar el destino de sus exportaciones}

A la luz de lo comentado anteriormente, se comprende que el segundo gran objetivo que persigue desde hace tiempo el Gobierno canadiense, con poco éxito hasta la fecha, ha sido diversificar sus relaciones comerciales para reducir la enorme dependencia que mantiene con EE. UU., a donde en 2019 se dirigieron el $75 \%$ de sus exportaciones. La vulnerabilidad que genera esta situación ha sido puesta de manifiesto en 2020 con ocasión de los cierres de actividad en Canadá y EE. UU. decretados para combatir la pandemia de la COVID-19, que han generado en el sector de la automoción una caída de las exportaciones bilaterales canadienses del $23,8 \%$ y de las importaciones bilaterales del 25,1\%. En el sector energético, las exportaciones bilaterales se contrajeron casi en un $30 \%$ y las importaciones un $37 \%$.

La principal herramienta para ampliar el número de socios comerciales ha sido la negociación de acuerdos comerciales. Canadá tiene en vigor catorce acuerdos comerciales que involucran a 51 países y a 1.500 millones de consumidores. Además del CUSMA ya mencionado, destacan: el Acuerdo Económico y Comercial Global (CETA) con la Unión Europea — que es objeto de otro artículo en esta publicación-, en vigor provisionalmente desde septiembre 2017; y el Acuerdo Transpacífico de Cooperación Económica (CPTPP), en vigor desde el 30 de diciembre 2018 para Australia, Canadá, Japón, México, Nueva Zelanda y Singapur, y desde el 15 de enero 2019 para Vietnam.

Hasta el momento, no parece que este último haya impulsado significativamente los flujos comerciales canadienses con sus países miembros. No obstante, es previsible que en el futuro esto cambie atendiendo al gran dinamismo económico que la región Asia-Pacífico está exhibiendo, así como a la próxima adhesión de Reino Unido, cuya negociación ya se ha iniciado.

Además de con las mencionadas zonas comerciales, Canadá tiene firmados acuerdos de libre comercio individuales con países como Chile, Perú, Colombia, Panamá, Israel, Jordania o Corea del Sur. 


\subsection{Principales socios comerciales}

Como ya se ha adelantado anteriormente, el resultado de la estrategia de diversificación no ha arrojado resultados muy optimistas hasta el momento y, en 2020 , el $73,38 \%$ de las exportaciones canadienses se dirigen a Estados Unidos, siendo la Unión Europea el segundo destinatario $(9,16 \%)$.

Por el lado de las importaciones canadienses, la concentración en Estados Unidos es menor y, de hecho, en 2020, las provenientes de EE. UU. han caído por debajo del $50 \%$, representando el $48 \%$. También en 2020, la UE (12,5\% de las importaciones que recibe Canadá) ha dejado de ser la segunda zona proveedora, dejando el puesto a China, que es origen del $14 \%$ de las importaciones.

En los últimos cinco años, los mayores déficits comerciales de Canadá fueron con China, México y Alemania, mientras que los mayores superávits comerciales se concentraron en Estados Unidos y Reino Unido.

\section{Mirando al futuro}

\subsection{Perspectivas favorables}

Precisamente por su grado de apertura y alta dependencia de los flujos comerciales, Canadá se ha visto muy afectado por las medidas de restricción de la actividad no esencial -incluido el cierre de la frontera desde marzo de 2020 hasta agosto de 2021 para los desplazamientos no esenciales desde EE. UU., y hasta septiembre de 2021 para los demás paísesadoptadas para frenar la pandemia. De hecho, el PIB se contrajo en 2020 un 5,3\%, la mayor caída desde 1961, fecha desde la que se publican datos comparables. Si consideramos los países del G-7, Canadá se ha visto menos afectado que Reino Unido, Italia o Francia, pero más que Alemania, Japón o Estados Unidos.

La pandemia ha tenido también un profundo impacto en el mercado laboral, pasando la tasa anual de desempleo del 5,7\% en 2019 al 9,5\% en 2020. La recuperación del empleo ha avanzado al compás de los diversos cierres y reaperturas de la actividad económica no esencial, y no ha alcanzado el nivel de empleo prepandemia en el primer semestre de 2021.

Es importante destacar el papel que los estímulos fiscales y monetarios han jugado, y siguen jugando, en mitigar la contracción de la actividad económica. Efectivamente, a nivel monetario, el Banco de Canadá bajó el tipo de interés hasta el 0,25\%, nivel en el que se mantiene desde marzo de 2020 y en el que continuará hasta que desaparezca el output gap, previsiblemente en la segunda mitad de 2022. Lo anterior se acompañó de potentes medidas de liquidez para el conjunto de la economía.

A nivel fiscal, el Gobierno federal adoptó diversas medidas como transferencias de emergencia, subsidios, diferimiento de obligaciones impositivas y créditos a personas físicas y empresas equivalentes al $15,4 \%$ del PIB, que alcanzan el $18,6 \%$ cuando se toman en consideración las medidas aprobadas por las provincias.

Si bien la recuperación en el primer semestre de 2021 ha sido menos robusta de lo esperado - debido al mantenimiento de las medidas de cierre de la actividad no esencial durante un plazo muy dilatado, así como a ciertas disrupciones en las cadenas de suministro-, las perspectivas son favorables y, según las últimas estimaciones del Banco de Canadá de julio 2021, la economía crecerá un $6 \%$ este año, moderándose el crecimiento hasta el $4,6 \%$ en 2022 y $3,3 \%$ en 2023. 
Entre los factores que explicarían esta proyección se encuentra el fuerte impulso que recibió la campaña de vacunación a partir de mayo, que logró que a mediados de agosto más del $82 \%$ de la población por encima de doce años hubiera recibido al menos una dosis de la vacuna anti-COVID-19 y que casi el $75 \%$ de dicha población estuviera completamente vacunada $^{2}$, reduciendo así significativamente la incertidumbre asociada a la pandemia y mejorando la confianza de empresas y consumidores. En este marco, el Banco de Canadá espera que la demanda retenida y el incremento del ahorro impulsen notablemente el consumo en la segunda mitad del año. Por su parte, el crecimiento robusto de la demanda extranjera -en particular la estadounidense - y los precios altos de las materias primas contribuirán a impulsar las exportaciones y la inversión empresarial.

Adicionalmente a este rebote esperado para la segunda mitad del año, las perspectivas del crecimiento a más largo plazo vendrán marcadas por la capacidad de Canadá para responder a los principales desafíos que tiene por delante e incrementar su productividad y su output potencial.

\subsection{Un mercado fragmentado}

Uno de los problemas que lastra tradicionalmente la competitividad del país es la ausencia de un mercado interior. Un estudio del $\mathrm{FMI}^{3}$, publicado en 2019, concluye que la eliminación de todas las barreras comerciales no geográficas incrementaría los flujos de intercambio internos al nivel de los internacionales y aumentaría el

https://health-infobase.canada.ca/covid-19/vaccination-coverage/ Internal Trade in Canada: Case for liberalization.
PIB real per cápita en un $4 \%$, logrando ganancias de productividad en virtud de los desplazamientos de trabajadores de las zonas con menor productividad media (Columbia Británica, Alberta y Ontario) a las demás regiones.

El mencionado estudio distingue cuatro tipos de barreras que entorpecen el comercio interprovincial: las barreras naturales derivadas de la enormidad del territorio y de características geográficas; las barreras prohibitivas contenidas en leyes provinciales que impiden el comercio interno, por ejemplo para bebidas alcohólicas; las barreras técnicas que surgen de las diversas regulaciones sectoriales que difieren en cada provincia; y las barreras administrativas relacionados con licencias, registros y otros requisitos exigidos para operar en cada provincia.

A diferencia de la estrategia de comercio internacional mencionada anteriormente, plasmada en numerosos acuerdos comerciales, los diversos intentos de reducir las barreras internas al comercio no han logrado avanzar decididamente hacia un auténtico mercado interior. Uno de los primeros intentos fue, en 1995, la firma de un acuerdo sobre comercio interno que no logró apreciables resultados debido a un enfoque poco ambicioso basado en una lista positiva de sectores cubiertos por el acuerdo y mecanismos débiles de resolución de conflictos.

Prueba de los escasos logros es que si a principios de la década de 1980 tanto el comercio internacional como el interprovincial suponían el 55\% del $\mathrm{PIB}^{4}$, en 2017 el comercio internacional suponía el $65 \%^{5}$ y el interno el $40 \%$ del PIB.

\footnotetext{
4 La suma de exportaciones e importaciones en relación con el PIB.

5 Este porcentaje se elevó hasta el $80 \%$ en la década de 1990, tras la firma del Acuerdo de Libre Comercio entre Canadá y Estados Unidos y tras la firma del NAFTA.
} 
Dirigido a evitar que las empresas extranjeras de países signatarios de acuerdos comerciales con Canadá —en particular las europeas - tuvieran mejor acceso al mercado canadiense que las empresas locales, el 1 de julio de 2017 el Gobierno federal, junto con las provincias y territorios, firmó un nuevo acuerdo de comercio interior basado en un enfoque de lista negativa (aunque ocupando las excepciones 135 páginas), mejoras en los mecanismos de solución de disputas y cobertura de contratación pública y en la promoción de la cooperación regulatoria.

A pesar de ciertos avances recientes hacia una mayor integración regional, Canadá sigue estando fragmentado en los siguientes mercados regionales:

- Las provincias del Atlántico (Nuevo Brunswick, Nueva Escocia, Terranova y Labrador, y la Isla del Príncipe Eduardo) mantienen estrechas relaciones comerciales con el nordeste de EE. UU. Su población de 2,4 millones de habitantes aporta del orden del $6 \%$ del PIB con una actividad económica basada en los recursos naturales (pesca, energía y minerales), aunque diversificada recientemente hacia nuevos sectores como las telecomunicaciones, la biotecnología y las industrias medioambientales.

- La provincia de Quebec -la segunda por número de habitantes (8,57 millones) y por contribución al PIB (19\% del total nacional) - es mayoritariamente francófona y se extiende sobre más de 1,5 millones de $\mathrm{km}^{2}$. Posee abundantes recursos naturales y energéticos y dispone de una industria muy desarrollada, con una especialización sectorial marcada por la industria aeroespacial, las telecomunicaciones, la industria farmacéutica y la biotecnología.

- Ontario es la provincia más potente, con 14,74 millones de habitantes y una aportación del $37 \%$ al PIB. Destacan los sectores de automoción, acero, química industrial, aeronáutica, agroalimentación, software y telecomunicaciones. La provincia es también uno de los principales ejes del tráfico de mercancías entre Canadá y Estados Unidos.

- Las provincias de las Praderas (Manitoba, Saskatchewan y Alberta) tienen una superficie de 2 millones de $\mathrm{km}^{2}$, una población de 6,91 millones de habitantes y una actividad económica —centrada en la agricultura, la minería y los hidrocarburos-que representa un $25 \%$ del PIB.

- Columbia Británica, con una población de 5,14 millones de habitantes y una aportación del orden del $12 \%$ del PIB, concentra su actividad en el sector maderero, la producción de pasta de papel y la energía. También han ido adquiriendo importancia la electrónica, el software, la biotecnología y las tecnologías medioambientales. Es destacable el crecimiento en los últimos años de la relación de la provincia con Asia.

- Los Territorios (Territorios del Noroeste, Yukón y Nunavut) ocupan buena parte de las regiones árticas de Canadá y están escasamente poblados.

\subsection{Impulsar la competencia}

El segundo de los desafíos que tiene por delante Canadá para incrementar su productividad es reforzar la competencia en determinados sectores clave. En efecto, Canadá $D$ 
mantiene estrictas limitaciones a la participación de capital extranjero en los sectores de telecomunicaciones, banca, transporte aéreo e industria cultural. Asimismo, los productos lácteos, aves de corral y huevos están sujetos a regulación de la oferta para asegurar que la demanda interna se corresponda con la producción y las importaciones reguladas. En estos casos, el acceso a los mercados se otorga mediante contingentes arancelarios fijados en el marco de la OMC, así como en algunos acuerdos comerciales suscritos por Canadá como el CETA.

Para dar una idea del grado de proteccionismo, podemos recurrir al indicador de la OCDE que mide el nivel de barreras de acceso a mercado y de competencia (PMR ${ }^{6}$, por sus siglas en inglés), que atribuye una puntuación entre 0 y 5 , reflejando cuanto más baja sea esta, menor intervencionismo público y mayor competencia. Pues bien, frente a una media OCDE de 1,40, Canadá presenta un índice de 1,73. A mayor abundamiento, para el subindicador de barreras al comercio y la inversión -en el que la media de países OCDE se sitúa en 0,69 y España obtiene una puntuación de 0,48-, Canadá presenta una puntuación de 1,01, solo superada por Chile, Israel, Corea del Sur y México.

De igual manera, cabe destacar que, según un informe del Fraser Institute ${ }^{7}$, publicado el 23 de mayo de 2019 , aproximadamente un $22 \%$ de la economía canadiense se encuentra protegida de la competencia a través de restricciones a la inversión extranjera y de los monopolios públicos $^{8}$, siendo los sectores con mayores barreras a la entrada el transporte aéreo, las

\footnotetext{
6 https://www.oecd.org/economy/reform/indicators-of-product-marketregulation/

7 https://www.fraserinstitute.org/studies/walled-from-competition-measuringprotected-industries-in-canada

8 A modo de ejemplo, se encuentran protegidos de la competencia los monopolios provinciales de distribución minorista de bebidas alcohólicas, la distribución de energía, el tránsito urbano o Canada Post, que monopoliza el mercado interno de envío de cartas.
}

telecomunicaciones y la radio y televisión. Dicho porcentaje se elevaría hasta el $35 \%$ si incluyéramos las profesiones reguladas y las barreras interprovinciales a la competencia.

\subsection{Hacia un nuevo modelo energético}

Otro de los factores que va a condicionar la evolución económica de Canadá es la gran transformación energética a la que se enfrenta el país para cumplir con el ambicioso objetivo de alcanzar, en 2050, cero emisiones netas de gases de efecto invernadero. La ley que recoge este compromiso fue aprobada antes del verano y establece metas de reducción de emisiones cada cinco años entre 2030 y 2050 . El objetivo para 2030 es reducir entre el 40 y el $45 \%$ los niveles de emisiones de 2005.

La estrategia de lucha contra el cambio climático de Canadá se estructura en torno a los siguientes elementos:

- El Gobierno federal fija un precio mínimo al carbono, que aumenta progresivamente hasta alcanzar 170 dólares canadienses por tonelada en $2030^{\circ}$ (unos 114 euros). Es importante subrayar que la Corte Suprema dictaminó, en marzo de 2021, que el mecanismo federal de precios de carbono es acorde a la Constitución. Las provincias tienen la flexibilidad de aplicarlo a través de un impuesto al carbono o de sustituirlo por un sistema equivalente, también progresivo, de comercio de emisiones ${ }^{10}$. Esta obligación cubre todas las emisiones, salvo las provenientes del sector forestal, agrícola y de residuos.

\footnotetext{
9 En 2019, el precio mínimo fue de 20 dólares por tonelada de $\mathrm{CO}_{2}$ y en 2021 fue de 40 dólares.

10 En este caso, las provincias tienen que cumplir con un objetivo de reducción de emisiones gradual hasta alcanzar en 2030 como mínimo el $30 \%$ del objetivo de reducción para Canadá.
} 
- Es importante destacar que, por ley, los ingresos provenientes de los diversos esquemas provinciales de precio de carbono permanecen en la jurisdicción de origen. La provincia o territorio cuyo sistema se adapta a las exigencias federales puede disponer de dichos ingresos. En el caso de Ontario, Manitoba, Alberta o Saskatchewan, donde se aplica un impuesto federal que adopta la forma de un recargo al combustible y a la calefacción, el Gobierno federal devuelve aproximadamente el $90 \%$ de los ingresos directamente a los contribuyentes a través de créditos fiscales ${ }^{11}$. El $10 \%$ restante se destina a proyectos de inversión en energías limpias. Adicionalmente al recargo, los emisores industriales se ven sometidos a un esquema de compraventa de créditos, que proporciona incentivos a reducir las emisiones manteniendo la competitividad.

- Incentivos federales sectoriales para la adquisición de vehículos cero emisiones, generadores eléctricos y para el reacondicionamiento de edificios.

- Reducción progresiva del máximo de $\mathrm{CO}_{2}$ permitido en las emisiones de los vehículos.

- Apoyo público a proyectos de inversión bajos en carbono.

- Cabe señalar, por último, que Canadá está siguiendo muy de cerca la iniciativa de la Unión Europea de introducir ajustes en frontera por carbono, por lo que, en el medio plazo, no es descartable la aprobación de algún tipo de esquema en este sentido.

11 https://www.canada.ca/en/environment-climate-change/services/ climate-change/pricing-pollution-how-it-will-work.html
Lo anterior constituye un enorme desafío para Canadá, país que en 2018 emitió 729 millones de toneladas de gases de efecto invernadero, el $74 \%$ de los cuales provinieron de los combustibles fósiles. Hay que tener presente que el petróleo obtenido de las arenas bituminosas de Alberta genera entre 3 y 5 veces más emisiones por barril equivalente de petróleo, según la consultora energética independiente Rystad Energy.

Adicionalmente, un estudio reciente publicado por el C.D. Howe Institute señala que, para alcanzar los objetivos medioambientales mencionados anteriormente, debe producirse una transformación completa del parque móvil, pasando los vehículos de cero emisiones del $3,5 \%$ del total de ventas en 2020 al $70-75 \%$ en 2030. Por ley, en 2035 , el $100 \%$ de las ventas de vehículos de pasajeros debe componerse de vehículos sin emisiones.

La financiación de la estrategia de lucha contra el cambio climático es acorde con lo ambicioso de los objetivos. Entre las diversas herramientas financieras destacan el Fondo para la Innovación Estratégica, que destinará $3.000 \mathrm{MCAD}^{12}$ (más de 2.000 millones de euros) en cinco años para impulsar la transformación industrial hacia soluciones limpias; 2.600 MCAD en siete años para eficiencia energética en edificios residenciales; 2.000 MCAD para reacondicionar edificios comerciales; cerca de 1.000 MCAD para proyectos de energía renovable y redes; 150 MCAD en tres años para estaciones de carga para vehículos sin emisiones; 1.500 MCAD para la adquisición de autobuses sin emisiones; 1.500 MCAD para producción y utilización de combustible bajo en carbono como hidrógeno; más de 3.000 MCAD para proyectos de reforestación.

12 Millones de dólares canadienses. 


\subsection{El reto de mejorar las infraestructuras}

Finalmente, otro de los grandes desafíos que enfrenta Canadá y que reviste gran interés para las empresas españolas es la necesidad de desarrollar sus infraestructuras. Entre 1960 y 1975, las inversiones en infraestructuras en Canadá se situaron en la media en comparación con los países de la OCDE. Sin embargo, durante los años 1975-2005, marcados por la voluntad política de reducir el déficit público, Canadá realizó un esfuerzo inversor muy por debajo del que llevaron a cabo sus pares. A pesar del impulso económico que siguió a la crisis financiera de 2008, Canadá figura en la vigésima sexta posición en el indicador de calidad de las infraestructuras del último Índice Global de Competitividad ${ }^{13}$.

Según el último informe sobre el estado de las infraestructuras en Canadá, elaborado por la federación de municipios y diversas asociaciones sectoriales ${ }^{14}$, la mayoría de las infraestructuras que se utilizan a diario tiene una antigüedad superior a los veinte años, y entre el $10 \%$ y el $20 \%$ de los diversos activos se encuentra en condiciones deterioradas o muy deterioradas, sugiriendo que se encuentran muy cerca del final de su vida útil. Si incluimos la categoría de activos con algún signo de deterioro, el porcentaje de instalaciones que deben ser reemplazadas o requieren de algún tipo de intervención se eleva hasta el 30-50\% ${ }^{15}$.

Para hacer frente a esta situación, en los últimos años, Canadá ha venido impulsando el esfuerzo inversor. En 2016, el Gobierno federal

\footnotetext{
13 http://www3.weforum.org/docs/WEF_TheGlobalCompetitiveness Report2019.pdf

14 http://canadianinfrastructure.ca/en/index.html

15 Los porcentajes varían en función de los subsectores. Por ejemplo: en carreteras, $39 \%$; en túneles y puentes, $38,7 \%$; en agua potable, $27,3 \%$; en aguas residuales, $28,1 \%$; en gestión de aguas de tormentas, $30 \%$; en transporte público, $30 \%$.
}

lanzó un plan de inversiones ${ }^{16}$ que compromete más de 180.000 MCAD (unos 120.000 millones de euros) en doce años, centrándose en cinco áreas prioritarias: transporte público, infraestructura verde, infraestructura social, comercio y transporte, y comunidades rurales y del norte.

Transcurridos cinco años, el Gobierno federal ha invertido del orden de 71.000 MCAD y ha aprobado más de 2.800 proyectos. A estos montos hay que sumar las aportaciones de provincias y municipios.

Finalmente, merece la pena destacar el lanzamiento, en abril de 2021, de una evaluación nacional de las infraestructuras ${ }^{17}$ que, inspirada en las llevadas a cabo por Australia en 2015 y Reino Unido en 2018, busca identificar las necesidades y prioridades en materia de infraestructuras que mejor contribuyan a incrementar la productividad y el crecimiento a largo plazo, a impulsar la transición hacia una economía sin emisiones y a aumentar la resiliencia de las instalaciones frente al cambio climático. Dicha evaluación será el primer paso hacia una planificación de los proyectos prioritarios de aquí a 2050 y, previsiblemente, incluirá la creación de un organismo independiente que los impulse.

\section{Conclusión}

Aún es pronto para calibrar el impacto de la pandemia de la COVID-19 en la actividad económica de Canadá. El optimismo que rodeaba la recuperación esperada en 2021 se ha visto truncado por un crecimiento anualizado en el segundo trimestre de $-1,1 \%$ (se estimaba un crecimiento del $2,5 \%$ ), que rompe con los $D$

\footnotetext{
16 https://www.infrastructure.gc.ca/plan/about-invest-apropos-eng.

https://www.infrastructure.gc.ca/nia-eni/nia-eni-eng.html
} $\mathrm{html}$ 
crecimientos positivos de los tres trimestres anteriores. Según los analistas, el impulso recibido desde el lado de la demanda, tras el gasto retenido por meses, se ha traducido en presiones inflacionistas en un contexto marcado por limitaciones de la oferta, fruto de disrupciones en las cadenas de suministro.

En cualquier caso, los grandes avances logrados en el proceso de vacunación, junto con nuevos impulsos desde el lado de la demanda, apuntan a previsiones de crecimiento del PIB en el entorno del 5-6 \% para este año.

Recientemente la inflación ha sobrepasado el objetivo fijado por el Banco de Canadá del $2 \%$ y supera el $3 \%$. No obstante, hasta el momento, los comunicados de esta institución consideran que el incremento de precios será pasajero, por lo que, salvo nuevos aumentos abruptos, se prevé que el tipo de interés se mantenga este año en entornos muy bajos, contribuyendo así a impulsar la demanda y la inversión.

Este contexto económico propicio, junto con la apertura de la frontera a partir de septiembre 2021, incrementa el atractivo del mercado canadiense para las empresas españolas exportadoras, en particular para las proveedoras de productos agroalimentarios y de equipos y suministros para la construcción, así como las que ofrecen bienes y servicios relacionados con los grandes retos a los que se enfrenta el país y que los distintos Gobiernos van a priorizar en el medio plazo: infraestructuras, energía y medioambiente.

\section{Bibliografía}

Alvarez, J., Krznar, I., y Tombe, T. (2019). Internal Trade in Canada: Case for liberalization (IMF Working Papers). International Monetary Fund. https://www.imf.org/en/Publications/WP/ Issues/2019/07/22/Internal-Trade-in-CanadaCase-for-Liberalization-47100
Balyk, J., Livingston, B., Hastings-Simon, S., y Bishop, G. (2021). Driving Ambitions: The Implications of Decarbonizing the Transportation Sector by 2030 (Commentary No. 604, July 2021). C.D. Howe Institute. https://www.cdhowe.org/ public-policy-research/driving-ambitionsimplications-decarbonizing-transportation-sector-2030

Bank of Canada. (2021). Monetary policy report July 2021. https://www.bankofcanada.ca/wp-content/ uploads/2021/07/mpr-2021-07-14.pdf

Boothe, P., Chang, B., y Moloney, D. (2020). Canada's Manufacturing Sector: A Decade in Review. Trillium Network for Advanced Manufacturing. https://trilliummfg.ca/wp-content/uploads/ 2020/08/Trillium_CanadasManufacturingSector-ADecadeinReview-August2020_FA-justify.pdf

Canada Energy Regulator. (2021). Canadian crude oil exports: A 30 year review. https://www.cer-rec. gc.ca/en/data-analysis/energy-commodities/crude-oil-petroleum-products/report/canadiancrude-oil-exports-30-year-review/index.html

Geloso, V. (2019). Walled from competition: Measuring protected industries in Canada. Fraser Institute. https://www.fraserinstitute.org/sites/default/ files/walled-from-competition-measuring-protected-industries-in-canada.pdf

Global Affairs Canada. (2021). Canada's State of Trade 2021 - A closer Look at Foreign Direct Investment. Office of the Chief Economist. Government of Canada. https://www.international.gc.ca/ transparency-transparence/state-trade-commerce-international/2021.aspx?lang=eng\#a1

Infrastructure Canada. (2021). Building the Canada We Want in 2050: Engagement Paper on the National Infrastructure Assessment. https://www.infrastructure.gc.ca/nia-eni/nia-eni-eng.html

International Monetary Fund. (2021). IMF Country Report $n^{\circ}$ 21/55. Canada. Selected issues. https://www.imf.org/en/Publications/CR/ Issues/2021/03/17/Canada-Selected-Issues50274

Jiang, K. (2021). Annual trade report: Highlights of Canada's merchandise Trade Performance in 2020. Global Affairs Canada. Government of $D$ 
Canada. https://www.international.gc.ca/trade-commerce/economist-economiste/analysis-analyse/merchandise_trade-commerce_ marchandises.aspx?lang=eng

Marshall, B. (2021). The State of Canada's Mining Industry: Facts \& Figures 2020. The Mining Association of Canada. https://mining.ca/wp-content/uploads/2021/02/FF-2020-EN-Web-1.pdf

Natural Resources Canada. (2020). Energy fact book 2020-21. https://www.nrcan.gc.ca/sites/nrcan/files/energy/energy_fact/energy-factbook2020-2021-English.pdf
OMC. (2019). Trade policy review report by the Secretariat Canada. https://www.wto.org/english/tratop_e/tpr_e/s389_e.pdf

Statista. (2020). Insurance industry in Canada. https://www.statista.com/study/36873/insuranceindustry-in-canada-statista-dossier/

Sweeney, B.A. (2020). Canada's Automotive Industry: A Decade In Review. Trillium Network for Advanced Manufacturing. https://trilliummfg. ca/wp-content/uploads/2020/05/TrilliumReport_ Autolndustry-DecadeInReview-May2020_ 2A.pdf 
